\title{
Factors That Influence the Observed Fast Fragmentation of Peptides in Matrix-Assisted Laser Desorption
}

\author{
Robert S. Brown, Bryan L. Carr, and John J. Lennon \\ Department of Chemistry and Biochemistry, Utah State University, Logan, Utah, USA
}

\begin{abstract}
Fragmentation processes that occur very early during matrix-assisted laser desorption ionization (MALDI) of peptides are examined by utilization of delayed pulsed ion extraction with a linear time-of-flight mass spectrometer. The oxidized B chain of bovine insulin (MW $=3495.95$ $\mathrm{u}$ ), which produces a wide range of fragment ions, is utilized as a probe to examine the effects of several experimental parameters on this process. Experimental evidence suggests that this MALDI process is not prompt fragmentation and involves metastable ion decay that is quite different from that which is observed with postsource decay experiments. This conclusion is based upon the significant differences observed in the fragmentation products produced by the two techniques. This metastable ion decay process also appears to be over within the minimum pulse delay period ( $320 \mathrm{~ns}$ ) that is possible with the current pulsed ion extraction hardware. These two observations suggest that either different activation processes are involved in the two techniques or that the much different time frame of the methods influences the observed ion decay pathways. This fast MALDI metastable ion fragmentation also is shown to be influenced by both the MALDI matrix and the laser fluence. (J Am Soc Mass Spectrom 1996, 7, 225-232)
\end{abstract}

S ince its introduction, matrix-assisted laser desorption ionization (MALDI) has been considered primarily as a "soft" ionization process that produces almost exclusively intact protonated molecular analyte ion species [1-4]. In recent years, however, this early understanding of the MALDI process has been revised to take into account the significant degree of metastable ion behavior that has been observed by researchers utilizing reflectron-based time-of-flight (TOF) mass spectrometers $[5,6]$, sector mass spectrometers [7], and ion deceleration stages [8]. For peptide and protein analytes, this metastable ion decay produces ion fragments due to both small neutral molecule losses $\left(\mathrm{H}_{2} \mathrm{O}\right.$ and $\left.\mathrm{NH}_{3}\right)$ [7] and various peptide bond cleavages [9].

Although not normally a concern with linear TOF mass spectrometers, metastable ion decay can cause serious degradation of the mass resolution obtainable with ion mirror-based time-of-flight (TOF) mass spectrometers [6]. For a typical MALDI TOF mass spectrometer that utilizes continuous ion extraction, metastable ion decay, which occurs in the source during ion acceleration, results in incoherent ion signals that contribute to background noise in the TOF spectra. Longer time frame metastable ion decay that oc-

Addresss reprint request to Dr. Robert S. Brown, Department of Chemistry and Biochemistry, UMC 0300, Logan, UT 84322-0300. curs in the field-free region of a reflectron mass spectrometer results in improperly focused ions. This ion defocusing in the reflectron is due to the decreased kinetic energy of the decay ions relative to the stable precursor ions. For small molecule losses, this is exhibited as fronting on the precursor ion peak and can result in serious mass resolution losses. This is particularly problematic in the case of larger peptide-protein analytes, which appear to be more susceptible to substantial metastable ion decay [6]. This metastable ion decay phenomenon also may be responsible for the poor MALDI results obtained with larger oligonucleotide samples [10].

Although prompt fragmentation can be observed with MALDI in a limited number of examples of especially labile molecules [11, 12], the majority of the ion fragmentation that is observed in reflectron-based time-of-flight (TOF) mass spectrometers occurs after the ions have been accelerated out of the ion source in the field-free region prior to the ion mirror (presumably on the long microsecond time scale). This so-called postsource decay (PSD) phenomenon may be due to a combination of collisional activation of the molecules (bimolecular process) during their continuous extraction through the initial dense plume of desorbed species $[9,13]$ and to unimolecular ion decay associated with excess energy imparted to the ions during the ionization stage of the MALDI process [6]. This unimolecular activation has been suggested to be due 
to excess energy deposited in the analyte by the proton transfer reaction early in the desorption process.

Although PSD of MALDI-generated ions can be a problem in terms of lower mass resolution in time-offlight mass spectrometry and for long term ion stabilities necessary for the various ion trapping mass spectrometers [14, 15], it has proven useful in the characterization of the primary structures of smaller peptides [9]. By utilizing the ion mirror of a reflectron TOF mass spectrometer as an energy compensating device [16, 17] it is possible to scan the range of ion energies that are properly focused at the detector. This allows the generation of a composite TOF spectrum of the metastable fragment ions that result from the PSD of precursor ions. Such studies on a variety of peptides have shown that PSD ions are the result of common amide bond cleavage reactions similar to what is observed [9] in low energy collisional activation spectra of peptides.

An alternative to ion mirror-based PSD experiments that can be used to study very early time frame MALDI metastable ion decay is the use of delayed ion extraction with a linear TOF mass spectrometer [18]. A variable ion extraction delay period provides the time necessary for fast metastable ion decay to occur in the source prior to ion acceleration. The decay period accessible by this approach is determined by the delayed ion extraction electronics employed and the initial forward velocity of the MALDI-generated ions coupled with the length of the ion source region where delayed ion extraction is performed. For the instrumentation employed for this work, the accessible time frame is between $320 \mathrm{~ns}$ and about $4 \mu \mathrm{s}$. Any metastable ion decay that occurs after the ion extraction delay period will either not be distinguishable from stable precursor ions (field-free metastable decay) or appear as incoherent background noise (metastable decay during ion acceleration). Unlike the PSD experiment, a single set of delayed ion extraction parameters produces a well focused metastable ion decay spectrum over a wide mass-to-charge ratio range. The potential analytical utility for the study of the primary structures of several common peptides and even some small proteins with this technique has been reported [19].

With conventional PSD ion fragmentation experiments, the relative role(s) of unimolecular and bimolecular processes on the PSD metastable ion fragmentation cannot be discerned. If one accepts the dense plume model $[9,20]$ for MALDI, the use of continuous ion extraction of MALDI-generated ions through this plume can be expected to lead to high collisional activation of the ions during their acceleration out of the ion source. Any unimolecular ion activation that also occurs due to the ionization process itself [6] cannot be distinguished in the resulting PSD spectra. By employing delayed ion extraction with a linear TOF mass spectrometer, the contribution of collisional processes to the fast metastable ion decay spectra can be minimized. The initial absence of an applied extraction field substantially limits the energy transferred by collisions between neutrals and ions during the fast metastable ion decay time frame. Any ion decay that results from collisional activation after the delay period when the extraction field is established in the source will not be observed as discrete metastable decay ions in a linear TOF instrument. By utilizing this approach, the contribution that unimolecular ion decay processes play early in the MALDI process can be established.

\section{Experimental}

The delayed ion extraction linear TOF mass spectrometer utilized in these studies has been described in detail elsewhere [18] and only the major points will be discussed here. A linear TOF mass spectrometer of conventional design [21] was equipped with a floating (up to $30-\mathrm{kV}$ ) high voltage pulser with the capability to produce up to a $3-k V$ fast rise time $(\approx 25-n s)$ voltage output pulse. This output pulse voltage is applied to the sample probe tip, which is mounted flush with the surface of the repeller electrode of a conventional three grid TOF source assembly. By optimal choice of the applied pulse voltage [18], a wide mass-to-charge ratio range of fast metastable decay ions can be studied in a single TOF spectrum. Under optimal conditions, a mass resolution of as high as 3500 [full width at halfmaximum (fwhm)] has been obtained for smaller peptides (melittin) by utilizing a 500-megasample/s (MS/s) transient digitizer and a $150-\mathrm{MHz}$ bandwidth amplifier (R. S. Brown, unpublished results). With the elevated laser fluences employed to generate good signal-to-noise fast metastable ion spectra, mass resolution for the resulting fragment ion species is typically around $400-800$.

A pulsed nitrogen laser (model PL2300, PTI Lasers, Princeton, NJ) that produces 600-ps (fwhm) laser pulses at $337.1 \mathrm{~nm}$ was used for all of the studies presented. The oxidized B chain of bovine insulin used in these studies was purchased from Sigma Chemical Company (St. Louis, MO) and was used as received without additional purification. To insure that all chain B fragment ions observed with delayed ion extraction were due to MALDI metastable ion behavior and not to impurities or solution degradation products, conventional continuous ion extraction TOF mass spectra under identical conditions were collected on the same linear TOF mass spectrometer. All MALDI matrices employed in these studies were purchased from Aldrich Chemical Company (Milwaukee, WI). Ferulic acid (4-hydroxy-3-methoxy cinnamic acid) was purified after receipt by sublimation followed by recrystallization from a $1: 1 \mathrm{v} / \mathrm{v}$ acetonitrile/water solution. All other matrices were used as received. Matrix solutions were prepared as approximately $10-\mathrm{mM}$ solutions in acetonitrile/water $(30 / 70 \mathrm{v} / \mathrm{v})$ to which $0.1 \%$ trifluoroacetic acid was added. Analyte solutions were prepared in distilled deionized water at a concentra- 
tion of $10 \mu \mathrm{M}$. Samples were deposited (0.5- $\mu \mathrm{L}$ analyte followed by $2.5 \mu \mathrm{L}$ of matrix) via a microsyringe onto a 3-mm-diameter stainless steel probe tip, mixed with the end of the syringe needle, and allowed to air evaporate.

Data were collected at $5 \mathrm{~ns} /$ point time resolution and the resulting TOF spectra (average of typically 50 laser pulses signal averaged) were mass calibrated via a standard [22] two point linear fit from two known masses (singly and doubly protonated oxidized B chain molecular ions) and their associated flight times. The often low level of peptide fragment ions produced under the MALDI conditions employed resulted in a dynamic range problem for the 8-bit transient digitizer (LeCroy, Chestnut Ridge, NY, model 8828) when higher laser fluences were employed. To characterize the lowest intensity fragment ion data with acceptable signalto-noise ratio, the gain of the amplifier could be increased such that the protonated molecular ion signal was not within the range of the transient digitizer input signal levels. This allowed identification of low level fragment ions but required an initial TOF spectrum to be taken with the protonated molecular ions "on scale" for mass calibration purposes.

Analyte photodegradation studies employed a model RPR-100 photochemical reactor (Southern New England Ultraviolet Co., Middletown, CT). This reactor produced approximately $336 \mathrm{~W}$ of UV irradiation from 16 lamps whose spectral output was centered at 300 $\mathrm{nm}$ and exhibited a fwhm of $\pm 30 \mathrm{~nm}$.

\section{Results and Discussion}

Figure 1 shows the suggested ion structures for the fast metastable ion decay species that have been observed with delayed ion extraction. The most facile bonds for cleavage in peptides involve the amide linkages between the amino acid residues. A systematic nomenclature [23] has been utilized to describe the ion species observed in collision-induced fragmentation mass spectra peptides. Via this nomenclature, PSD ions have been reported [9] to produce primarily ion series of the type $a_{n}, a_{n}-17, b_{n}$, and $b_{n}-17$ for a series of low to moderate mass peptides. Recently, similar ion series (primarily $a_{n}$ and $b_{n}$ ) have been reported [24] as the MALDI metastable ion decay species observed in

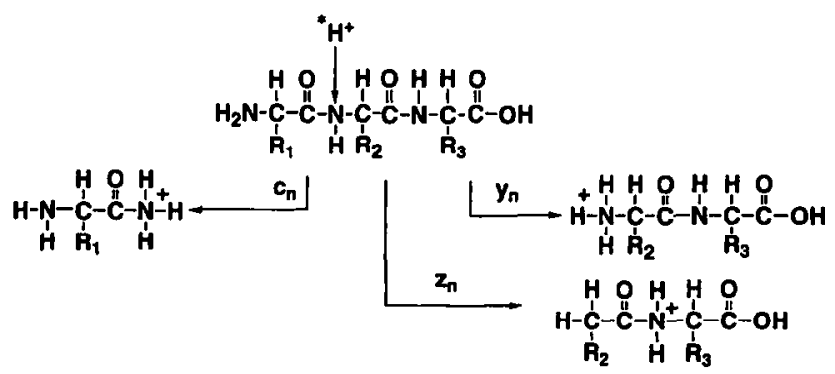

Figure 1. Possible structures for MALDI fast metastable ion fragments. an ion trap storage reflectron time-of-flight (IT/reTOF) instrument, which extends the time frame for metastable ion decay to $10-20 \mathrm{~ms}$.

The fast MALDI metastable ion decay products observed by delayed ion extraction result from very different bond cleavage reactions than those observed in PSD experiments. Although the exact ion fragmentation for a particular analyte is somewhat matrix dependent (see subsequent discussion), the positive ion fragmentation series that have been identified involve $c_{n^{-}}, y_{n^{-}}$, and $z_{n}$-type ions. The $z_{n}$-type ions observed in the fast metastable ion decay correspond to a $15-u$ mass difference relative to the $y_{n}$ ion series. This is in contrast to the 17-u difference for $z_{n}$-type ions observed in collisional activation tandem mass spectrometry. Such significant differences between the PSD and delayed ion extraction experiments are consistent with different ion decay pathways being dominant in the two experiments.

If unimolecular decay processes are responsible for the fragmentation observed by delayed ion extraction, a number of possible energy transfer processes could be involved. Although prompt photodissociation of peptides has been reported at much shorter wavelengths [25] and does produce fragmentation that is similar to that observed in the MALDI fast metastable ion decay spectra, the long UV wavelength $(337.1 \mathrm{~nm}$ ) and the low molar absorptivities of the analytes at this wavelength should prevent direct photon-induced dissociation. A more likely activation process for this fast metastable ion decay would seem to involve some initial energy transfer from a strongly absorbing desorption species (i.e., matrix molecules or matrix fragments) as has been proposed by other researchers [6].

\section{Matrix Influence on Fast Metastable Ion Fragmentation}

One factor that is known to influence the degree of PSD that is observed [6] is the MALDI matrix that is utilized. To examine the effect on the fast metastable ion decay of the MALDI matrix, a suitable probe analyte is needed. The oxidized B chain of bovine insulin was chosen as a representative model peptide. From earlier studies [19], this moderate sized peptide was observed to exhibit significant metastable ion decay on the necessary time scale.

Several common MALDI matrices were selected based upon differences in both their chemical structures and matrix characteristics. The 2,5-dihydroxy benzoic acid (DHB) matrix was known from previous studies [19] to provide the most general series of fragments for small to moderate sized peptides. Ferulic acid (4-hydroxy-3-methoxy cinnamic acid) and sinapinic acid (3,5-dimethoxy-4-hydroxy cinnamic acid) were chosen as two of the more widely utilized MALDI matrices. They also could provide a measure of how sensitive the fast MALDI metastable ion decay is to 
subtle structural changes. The high degree of PSD activation observed with $\alpha$-cyano-4-hydroxy cinnamic acid [6] made it a logical choice. The additional tendencies for this matrix to induce higher charge state ions and to produce lower matrix adduct ions [26] were also factors in its selection. The 4-hydroxy- $\alpha$ phenyl cinnamic acid matrix is included because it produces similar reduced matrix adduction, but no enhancement in multiple charging. Finally, the 7-hydroxy coumarin matrix was chosen for inclusion because of its (presumably) much different proton transfer process.

The fast metastable ion decay that was observed for the insulin B chain sample in these different matrices is summarized in Table 1 . The 10 most intense fragment ions that were observed for each matrix are noted in bold typeface. Given the differences in the MALDI properties of the matrices studied, it is somewhat surprising to note the general similarity of the fast MALDI fragment ions that are observed. In the majority of cases, only the relative intensities of the fragments change with the matrix. What does seem to change with the matrix is which particular amide residue is more susceptible to cleavage. Although the observed differences in the relative intensities of the fragment ions from matrix to matrix are often subtle, they are reproducible for a given set of experimental conditions. In an attempt to provide a measure of the reproducibility, three similar samples (insulin B chain in 2,4-dihydroxy benzophenone) were prepared and spectra were recorded for each from three different sample surfaces. By using the 10 most intense fragment ions (normalized as a percent of the doubly protonated molecular ion), the standard deviation in the intensity for each individual fragment ion was determined for the nine spectra. The average of the standard deviation for these fragment ions was $7 \%$.

Figures 2, 3, and 4 demonstrate some of the variations in the observed metastable fragmentation for different matrices. The DHB matrix (Figure 2) produces both intense $\mathrm{y}_{n^{-}}$and $c_{n}$-type fragment ions, which generally provide complementary sequences information for this peptide. The ferulic acid spectrum shown in Figure 3 exhibits fewer and often less intense $y_{n}$ fragments. Certain $y_{n}$ fragments, such as the large $y_{15}$ fragment (a leucine cleavage), are more intense.

Table 1. MALDI rapid metastable decay ions observed for the oxidized B chain of bovine insulin ( $\mathrm{MW}=3495.95)$ in various matrices ${ }^{\circ}$

\begin{tabular}{|c|c|c|c|c|c|c|c|c|c|c|c|c|c|c|c|c|c|}
\hline \multirow{2}{*}{$\frac{\text { Residue }^{\mathrm{b}}}{\text { Phe }}$} & \multicolumn{3}{|c|}{$2,5-\mathrm{DHB}^{\mathrm{c}}$} & \multicolumn{3}{|c|}{ Ferulic } & \multicolumn{3}{|c|}{ Sinapinic } & \multicolumn{3}{|c|}{ 4-HACCA } & \multicolumn{2}{|c|}{ 4-HAPCA } & \multicolumn{3}{|c|}{$7-\mathrm{HC}^{\prime}$} \\
\hline & $Y_{29}$ & $z_{29}$ & & $y_{29}$ & & & $Y_{29}$ & $z_{29}$ & & $V_{29}$ & $z_{29}$ & & $y_{29}$ & & $V_{29}$ & & \\
\hline Val & $Y_{28}$ & $z_{28}$ & & $Y_{28}$ & $z_{28}$ & & $Y_{28}$ & $z_{28}$ & & $V_{28}$ & $z_{28}$ & & & & $y_{28}$ & & \\
\hline Asn & $y_{27}$ & $z_{27}$ & & $y_{27}$ & $z_{27}$ & & $Y_{27}$ & $z_{27}$ & & $Y_{27}$ & $z_{27}$ & & $Y_{27}$ & & $Y_{27}$ & $z_{27}$ & \\
\hline Gln & $y_{26}$ & $z_{26}$ & & $y_{26}$ & $z_{26}$ & & $Y_{26}$ & $z_{26}$ & & $Y_{26}$ & $z_{26}$ & & & & $Y_{26}$ & & \\
\hline His & $y_{25}$ & $z_{25}$ & & $y_{25}$ & $z_{25}$ & & & $z_{25}$ & & $V_{25}$ & $z_{25}$ & & & & $V_{25}$ & & \\
\hline Leu & $y_{24}$ & $z_{24}$ & $\mathrm{c}_{5}$ & $Y_{24}$ & & & $V_{24}$ & $z_{24}$ & & $V_{24}$ & $z_{24}$ & & $y_{24}$ & & $y_{24}$ & & \\
\hline Cys & $y_{23}$ & $z_{23}$ & $c_{6}$ & $Y_{23}$ & & $\mathbf{c}_{6}$ & $\mathbf{Y}_{23}$ & & $c_{6}$ & $V_{23}$ & $z_{23}$ & & $y_{23}$ & & $Y_{23}$ & & $c_{6}$ \\
\hline Gly & $y_{22}$ & $z_{22}$ & $c_{7}$ & $V_{22}$ & $z_{22}$ & $c_{7}$ & $V_{22}$ & $z_{22}$ & & $V_{22}$ & $z_{22}$ & & $V_{22}$ & $c_{7}$ & $V_{22}$ & & \\
\hline Ser & $y_{21}$ & $z_{21}$ & $c_{8}$ & $y_{21}$ & $z_{21}$ & $c_{8}$ & $Y_{21}$ & $z_{21}$ & $c_{8}$ & $V_{21}$ & $z_{21}$ & & $Y_{21}$ & $c_{8}$ & $V_{21}$ & & $c_{8}$ \\
\hline His & $y_{20}$ & $z_{20}$ & $c_{\vartheta}$ & & & $c_{9}$ & $Y_{20}$ & & $c_{9}$ & $\mathrm{~V}_{20}$ & $\mathbf{z}_{20}$ & & $y_{20}$ & & $y_{20}$ & & \\
\hline Leu & $y_{19}$ & $z_{19}$ & $c_{10}$ & & & $c_{10}$ & $y_{19}$ & & $c_{10}$ & $Y_{19}$ & $z_{19}$ & & $Y_{19}$ & $c_{10}$ & $Y_{19}$ & & \\
\hline Val & $Y_{18}$ & $z_{18}$ & $c_{11}$ & $y_{18}$ & & $c_{11}$ & $Y_{18}$ & $z_{18}$ & $c_{11}$ & $y_{18}$ & $z_{18}$ & $c_{11}$ & $y_{18}$ & $c_{11}$ & $y_{18}$ & & $c_{11}$ \\
\hline Glu & $V_{17}$ & $z_{17}$ & $c_{12}$ & & & $c_{12}$ & $y_{17}$ & & $c_{12}$ & $y_{17}$ & $z_{17}$ & $c_{12}$ & $y_{17}$ & $c_{12}$ & $y_{17}$ & & $c_{12}$ \\
\hline Ala & $Y_{16}$ & $z_{16}$ & $c_{13}$ & $Y_{16}$ & $z_{16}$ & $c_{13}$ & $y_{16}$ & & $c_{13}$ & $Y_{16}$ & $z_{16}$ & $c_{13}$ & $V_{16}$ & $c_{13}$ & $\mathbf{Y}_{16}$ & & $c_{13}$ \\
\hline Leu & $Y_{15}$ & $z_{15}$ & $c_{14}$ & $y_{15}$ & & $c_{14}$ & $Y_{15}$ & & $c_{14}$ & $\mathbf{Y}_{15}$ & $z_{15}$ & $c_{14}$ & $\mathbf{Y}_{15}$ & $c_{14}$ & $Y_{15}$ & & $c_{14}$ \\
\hline Tyr & $Y_{14}$ & $z_{14}$ & $c_{15}$ & $Y_{14}$ & $z_{14}$ & $c_{15}$ & $\mathbf{y}_{14}$ & & $c_{15}$ & $y_{14}$ & $z_{14}$ & $c_{15}$ & $y_{14}$ & $c_{15}$ & $y_{14}$ & & $c_{15}$ \\
\hline Leu & $Y_{13}$ & $z_{13}$ & $c_{16}$ & & & $c_{16}$ & & & $c_{16}$ & $y_{13}$ & $\mathbf{z}_{13}$ & $c_{16}$ & $y_{13}$ & $c_{16}$ & $Y_{13}$ & & $c_{16}$ \\
\hline Val & $y_{12}$ & $z_{12}$ & $c_{17}$ & $y_{12}$ & & $c_{17}$ & $y_{12}$ & & $c_{17}$ & $y_{12}$ & $\mathbf{z}_{12}$ & $c_{17}$ & $Y_{12}$ & $c_{17}$ & $\mathbf{Y}_{12}$ & & \\
\hline Cys & $y_{11}$ & $z_{11}$ & $c_{18}$ & $y_{11}$ & $z_{11}$ & $c_{18}$ & $y_{11}$ & $z_{11}$ & $c_{18}$ & $y_{11}$ & $z_{11}$ & $c_{18}$ & $y_{11}$ & $\mathbf{C}_{18}$ & $Y_{11}$ & & $c_{18}$ \\
\hline Gly & $Y_{10}$ & $z_{10}$ & $c_{19}$ & & $z_{10}$ & $c_{19}$ & $y_{10}$ & $z_{10}$ & & $y_{10}$ & $z_{10}$ & & & $\mathrm{c}_{19}$ & $Y_{10}$ & $z_{10}$ & \\
\hline Glu & $y_{9}$ & $z_{9}$ & $c_{20}$ & $Y_{9}$ & & $c_{20}$ & $y_{9}$ & $z_{9}$ & $\mathrm{c}_{20}$ & $Y_{9}$ & $z_{9}$ & $c_{20}$ & $Y_{9}$ & $\mathrm{c}_{20}$ & $Y_{9}$ & $z_{9}$ & \\
\hline Arg & $y_{8}$ & $z_{a}$ & $c_{21}$ & $Y_{8}$ & & $c_{21}$ & & $z_{8}$ & $c_{21}$ & & $\mathrm{z}_{\mathrm{g}}$ & & $V_{8}$ & & $Y_{8}$ & $z_{8}$ & \\
\hline Gly & $y_{7}$ & $z_{7}$ & $c_{22}$ & $y_{7}$ & & $c_{22}$ & $y_{7}$ & $z_{7}$ & $\mathrm{c}_{22}$ & & & $c_{22}$ & & $c_{22}$ & $Y_{7}$ & $z_{7}$ & \\
\hline Phe & $Y_{6}$ & $z_{6}$ & $c_{23}$ & $y_{6}$ & & $c_{23}$ & & $z_{6}$ & $\mathbf{c}_{23}$ & & & $c_{23}$ & & $c_{23}$ & $Y_{6}$ & & $c_{23}$ \\
\hline Phe & & & $c_{24}$ & & & $c_{24}$ & & & $\mathrm{c}_{24}$ & & & $c_{24}$ & & $\mathbf{c}_{24}$ & & & $c_{24}$ \\
\hline Tyr & & & $c_{25}$ & & & $c_{25}$ & & & $\mathbf{c}_{25}$ & & . & $c_{25}$ & & & & & \\
\hline $\begin{array}{l}\text { Thr } \\
\text { Pro }\end{array}$ & & & $c_{26}$ & & & $c_{26}$ & & & $c_{26}$ & & & $c_{26}$ & & & & & \\
\hline Lys & & & $c_{28}$ & & & $c_{28}$ & & & $c_{28}$ & & & & & & & & $c_{28}$ \\
\hline Ala & & & $c_{29}$ & & & $c_{29}$ & & & $c_{29}$ & & & $c_{29}$ & & $c_{29}$ & & & $\mathbf{c}_{29}$ \\
\hline
\end{tabular}




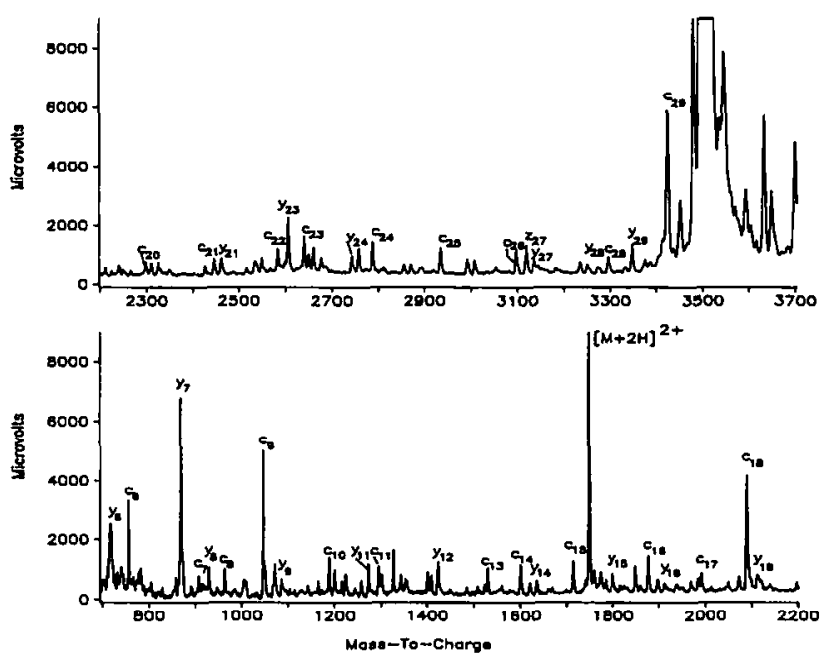

Figure 2. MALDI fast metastable ion decay spectrum of the oxidized B chain of bovine insulin obtained with delayed ion extraction. 2,5-dihydroxy benzoic acid matrix, 540-ns extraction delay, $1.50-\mathrm{kV}$ pulsed extraction voltage, and $24-\mathrm{kV}$ ion source bias voltage.

The greatest differences (although not dramatic) in the metastable ion fragments were observed with the weakly acidic 7-hydroxy coumarin (Figure 4). Instead of a strong $c_{n}$ fragment ion series as observed with the aromatic carboxylic acid-based matrices, the $\mathrm{y}_{n}$ fragment ion series are dominant and the $c_{n}$ fragment ion series is weaker and less complete. The intense ions at low mass-to-charge ratio in Figures 3 and 4 denoted with an asterisk are due to the matrix.

From our studies to date, it appears that for all MALDI matrices that produce strong protonated peptide ion signals, some fast metastable ion decay occurs. This fact, along with the differences in fragmentation
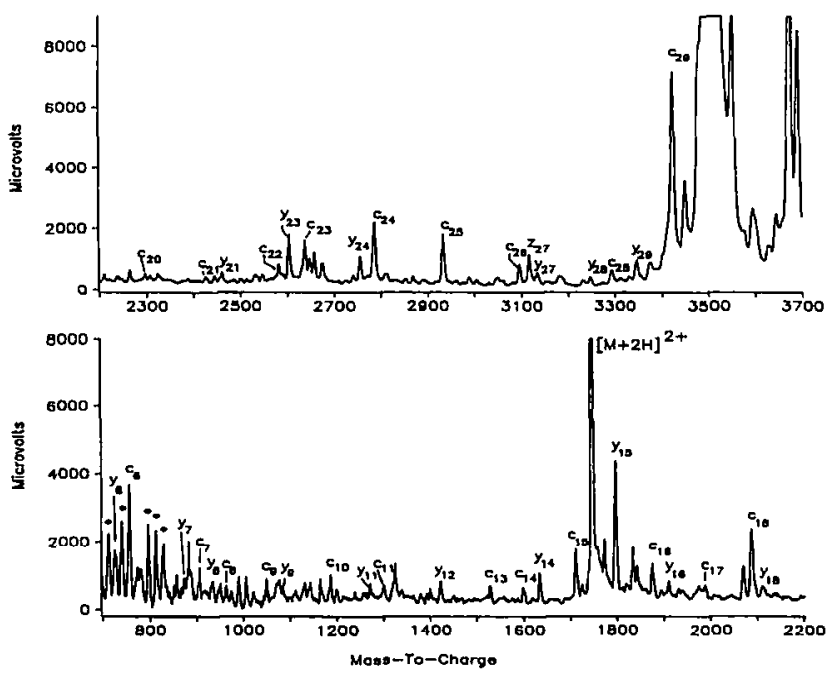

Figure 3. MALDI fast metastable ion decay spectrum of the oxidized B chain of bovine insulin obtained with delayed ion extraction. 4-hydroxy-3-methoxy cinnamic acid matrix, 540-ns extraction delay, $1.50-\mathrm{kV}$ pulsed extraction voltage, and $24-\mathrm{kV}$ ion source bias voltage.

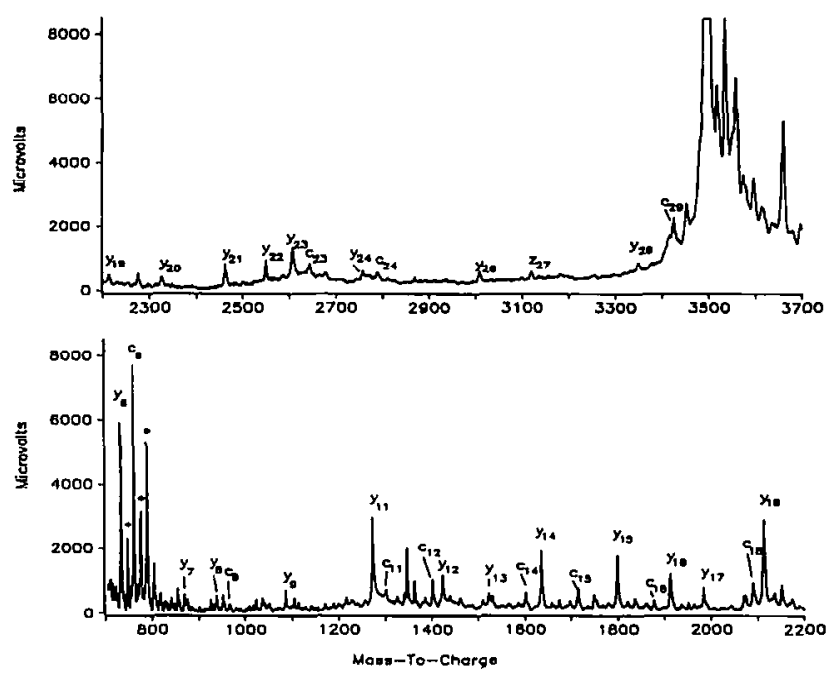

Figure 4. MALDI fast metastable ion decay spectrum of the oxidized $B$ chain of bovine insulin obtained with delayed ion extraction. 7-hydroxy coumarin matrix, 540-ns extraction delay, $1.50-\mathrm{kV}$ pulsed extraction voltage, and $24-\mathrm{kV}$ ion source bias voltage.

observed for the weakly acidic 7-hydroxy coumarin matrix, may indicate that proton transfer plays an important part in fragmentation. If an exothermic proton transfer reaction [6] occurs at some point early in the desorption process between the analyte and a matrix molecule (or fragment), the resulting excess energy transferred to the analyte during protonation may result in bond cleavages that are localized to a protonation site (amide group). Additional support for the importance of a proton transfer to the fast metastable ion decay process involves cationized peptide ions. In the case of MALDI spectra of smaller peptides such as substance $\mathrm{P}$, cationization (e.g., $[\mathrm{M}+\mathrm{Na}]^{+}$) readily competes with protonation when the sample is doped with an appropriate salt. In peptide samples examined to date, which produce significant amounts of both cationized and protonated molecular ion species, no cationized fragment ion species have been observed.

\section{Extraction Delay and Laser Fluence Effects}

Figure 5 shows a typical MALDI fast (560-ns extraction delay) metastable ion decay spectrum of the oxidized B chain of bovine insulin obtained from a 2,5dihydroxy benzoic acid matrix near threshold irradiance. This spectrum demonstrates the low relative intensity of fragment ions generated at laser fluences typical of continuous ion extraction experiments. The most intense fragments observed are comparable in intensity to the matrix adduct ion species (i.e., ions with intact matrix or matrix fragments bound to the analyte). This can be compared to the spectrum in Figure 2 that was taken from the same sample (different surface) at elevated laser irradiance levels. The laser fluence was adjusted to produce a singly protonated molecular ion signal for the oxidized $B$ chain of 


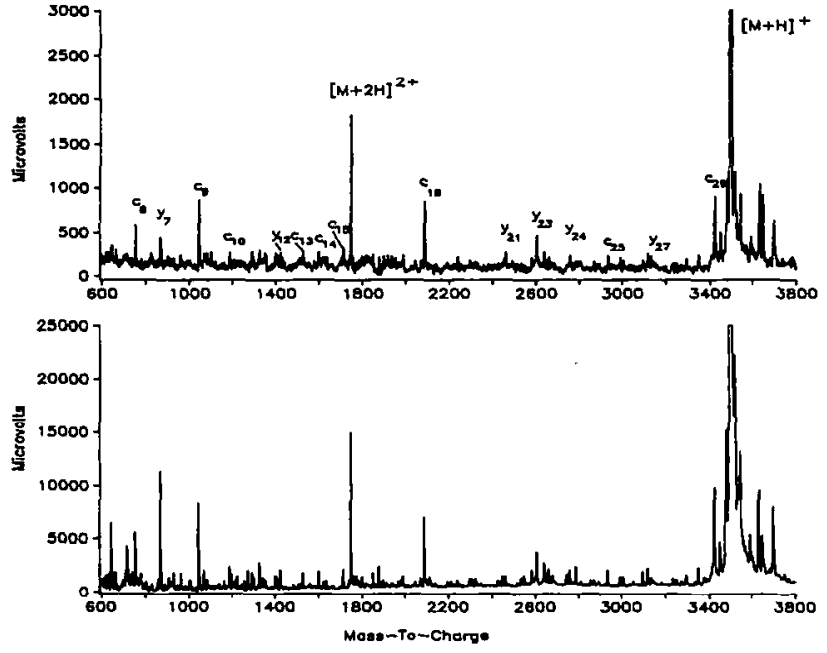

Figure 5. MALDI fast metastable ion decay spectrum of the oxidized B chain of bovine insulin obtained with delayed ion extraction near threshold irradiance. 2,5-dihydroxy benzoic acid matrix, $540-n s$ extraction delay, $1.50-\mathrm{kV}$ pulsed extraction voltage, and 24-kV ion source bias voltage.

bovine insulin that was approximately a factor of eight times larger than the signal obtained near threshold irradiance. Although the improvement in the signalto-noise ratio of the higher laser fluence spectrum is obvious, there is very little difference in the relative ion signals observed in the two spectra. This suggests that a very linear, first order decay process is involved in the case of 2,5-dihydroxy benzoic acid as the MALDI matrix. With the exception of an unusually facile bond cleavage ( $y_{11}$ ion) observed to increase at higher laser fluence when $\alpha$-cyano-4-hydroxy cinnamic acid matrix is used as a matrix (vide infra), similar results are observed for the other matrices studied.

A key question with regard to the nature of the metastable ion decay process in MALDI is what link, if any, exists between the short and long time frame metastable ion decay processes. Although the significantly different ion fragment series observed suggests fundamentally different ion activation processes are involved, do PSD-type fragment ions begin to appear at longer extraction delay times? To address this question, delayed extraction experiments were conducted within the time frame between $320 \mathrm{~ns}$ and $2 \mu$ s after the laser pulse. Results for the various common MALDI matrices that were examined are similar and only the results obtained with $\alpha$-cyano-4-hydroxy cinnamic acid will be presented.

Figure 6 shows three MALDI mass spectra produced by utilizing $\alpha$-cyano-4-hydroxy cinnamic acid as the matrix with continuous ion extraction (top) and delayed ion extraction at delay times of $350 \mathrm{~ns}$ (middle) and $1060 \mathrm{~ns}$ (bottom). The spectra were produced from different sample surfaces of the same sample preparation where the singly protonated molecular ion signals were of similar intensity $( \pm 5 \%)$. The same laser fluence (slightly above threshold irradiance) was
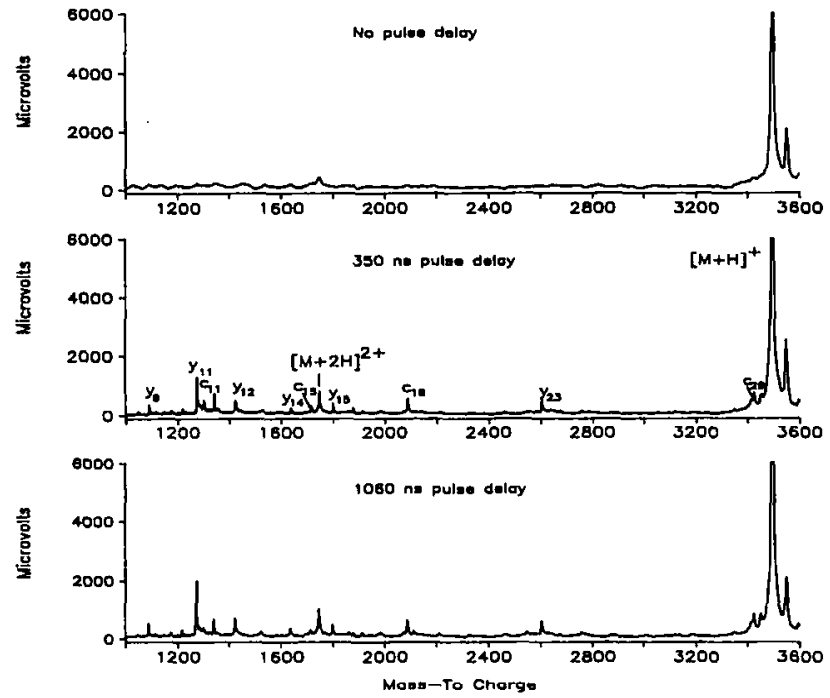

Figure 6. Effect of the ion extraction delay used on the yield of the fast metastable ion decay species from the oxidized B chain of bovine insulin produced by utilizing $\alpha$-cyano-4-hydroxy cinnamic acid as the matrix. Continuous ion extraction $(25-\mathrm{kV}$ source bias) spectrum (top), 350-ns extraction delay (middle), and 1060ns extraction delay (bottom).

utilized for each of the mass spectra. The spectra are plotted on the same vertical scale for comparison. No fragment ions are observed for the continuous ion extraction spectrum, which demonstrates that some finite period exists between ion formation and fragmentation. Although the delayed ion extraction experiment offers significant signal-to-noise enhancement over continuous ion extraction, this cannot explain the lack of observed fragment ions in the case of continuous ion extraction. The most intense fragment ion species observed (see also Figure 5) are clearly of similar intensity as the matrix adduct ion species that are observed well above the noise in continuous ion extraction experiments. These facts strongly suggest that the fragment ions observed are due to metastable ion decay.

The two delayed ion extraction spectra in Figure 6 are remarkably similar in terms of fragment ion types and intensities. A slight increase in the $y_{11}$ fragment (about $25 \%$ ) is the only significant difference observed at longer extraction delay. Significantly, no PSD-type ion fragments appear with increased delay. No significant changes in fragmentation with delay period were observed in similar experiments that used ferulic acid, sinapinic acid, and 2,5-dihydroxy benzoic acid. Within the experimental constraints of the reproducibility typical of MALDI; it appears that most, if not all, of the fast metastable ion decay is complete within the minimum delay period ( $320 \mathrm{~ns}$ ) possible with our current delayed extraction instrumentation.

The $y_{11}$ fragment ion that is observed to increase in Figure 6 as a function of the extraction delay time that is employed is an exception to the general observation that the fast fragmentation is complete very early 
( $<320 \mathrm{~ns})$ in the desorption event. The $y_{11}$ fragment ion appears to involve a very facile bond cleavage reaction when $\alpha$-cyano-4-hydroxy cinnamic acid is employed as the matrix. This intense fragment ion corresponds to cleavage at an oxidized cysteine residue in the bovine insulin B chain analyte. Although the two (oxidized) cysteine residues in the $B$ chain of bovine insulin normally are observed to be prominent bond cleavage sites with other matrices (see $c_{8}, c_{18}$, and $y_{23}$ fragments in Figure 2), the $\alpha$-cyano-4-hydroxy cinnamic acid matrix seems to induce more pronounced fragmentation at the $y_{11}$ site. This particular fragmentation also increases dramatically with the laser fluence for this matrix. By utilizing a fixed metastable ion decay period of 1060 ns, MALDI spectra (Figure 7) were collected from three different surfaces of a sample of the B chain of insulin prepared in $\alpha$-cyano-4hydroxy cinnamic acid. The laser fluence was adjusted for each spectrum so that increasing intensities $(1 \times$, $2 \times$ and $5 \times)$ of the singly protonated molecular ion species were produced.

The $y_{11}$ ion fragment is observed to increase in intensity to about $90 \%$ of the intensity of the singly protonated molecular ion species as the laser fluence is increased. Because each of the spectra in Figure 7 are scaled based upon the singly protonated molecular ion intensities in each spectrum, the apparent increase in the relative intensity of the other metastable fragment ions at the highest employed laser fluence is deceptive. Because the singly protonated molecular ion intensity is used to establish relative scaling between spectra, the significant degree of parent ion fragmentation affects the scaling in the high laser fluence spectrum. If the approximately equal intensities of the $y_{11}$ ion fragment and the singly protonated molecular ion are taken into account, the relative intensities of the frag-
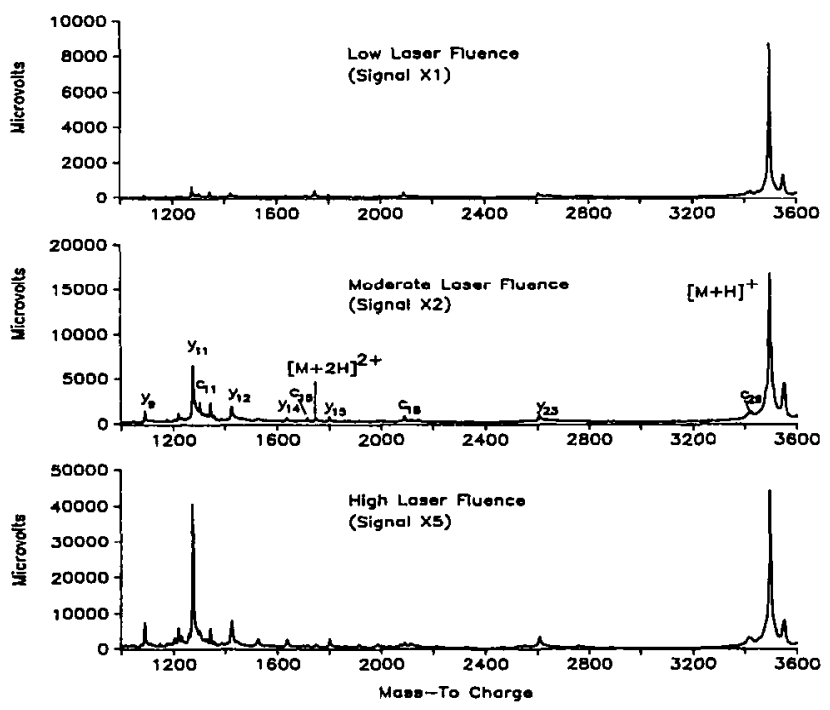

Figure 7. Effect of increased laser fluence with constant ion extraction delay (1060 ns) on the yield of fast metastable ion decay species from the oxidized $B$ chain of bovine insulin and $\alpha$-cyano-4-hydroxy cinnamic acid as the matrix. ment ions should be reduced by about a factor of 2 . This would make them similar in relative intensity (except for the $y_{11}$ ion) to what is observed at lower laser fluence. Such a dramatic increase in a single fragmentation pathway has only been observed to date with $\alpha$-cyano-4-hydroxy cinnamic acid. The reason for this unusually intense bond cleavage at the $y_{11}$ position with the oxidized $B$ chain of bovine insulin and $\alpha$-cyano-4-hydroxy cinnamic acid as the matrix is not explained easily. The strong laser fluence dependence suggests that some form of highly specific photon activation process may be occurring, but the nature of this process is not readily apparent.

Photon-induced sample degradation in the solid state prior to desorption could be argued, in lieu of metastable ion decay, as a possible source for some of the fragment ions observed with delayed ion extraction. However, given the significant intensities of some fragment ions even under near threshold laser irradiance, such solid state degradation products surely also would be observed in conventional continuous ion extraction MALDI experiments. Attempts to enhance the yield of insulin chain $B$ fast fragmentation by preirradiation of MALDI samples in a UV photoreactor yielded no increase in amide bond-related cleavages. Multiple disulfide bond cleavage reactions previously observed in the fast fragmentation MALDI spectra of porcine insulin (sinapinic acid matrix) [19] at levels comparable to $c_{n}, y_{n}$, and $z_{n}$ fragment ions were found to be enhanced greatly by UV irradiation in the photoreactor prior to MALDI. Although ion intensities for fragments that correspond to the reduced form of the individual $A$ and $B$ chain could be increased to almost the intensity of the singly protonated molecular ion (20-min irradiation), the only other fragmentation observed to increase was analyte decarboxylation. Similar disulfide cleavages of peptides have been reported in continuous ion extraction experiments $[27,28]$ and have been attributed to prompt fragmentation during desorption. These experiments suggest that disulfide bond cleavages and analyte decarboxylation observed in MALDI are due to photodegradation in the solid prior to desorption. The lack of increase in amide bond cleavages after photoirradiation suggests that $c_{n}, y_{n}$ and $z_{n}$ fragment ions observed in delayed extraction MALDI are not due to solid state photodegradation.

\section{Conclusions}

The large differences between the metastable ion fragments observed with reflectron PSD experiments and delayed ion extraction on a linear TOF experiments most probably are due to bimolecular collisional processes that dominate the PSD process. The low intensity fragmentation observed in the present studies with delayed ion extraction are consistent with a unimolecular ion activation process, possibly associated with the proton transfer step in MALDI. Similarities in the observed fragmentation with different matrices sug- 
gest a common process that provides similar degrees of ion activation. Additional studies with a wider range of matrices may provide additional insight into the fragmentation mechanisms that are involved.

\section{Acknowledgment}

The authors greatly acknowledge the assistance of Directed Energy, Inc. both for donation of the initial GRX 3.0K pulser and for helpful discussions with the staff concerning its use for this project. This research was supported, in part, by grants from the National Institutes of Health, Divisions of Research Resources (RR05311) and General Medical (GM47914) and with funds provided by Utah State University.

\section{References}

1. Karas, M.; Ingendoh, A.; Bahr, U.; Hillenkamp, F. Biomed. Environ. Mass Spectrom. 1989, 18, 841.

2. Karas, M.; Bahr, U.; Ingendoh, A.; Nordhoff, E.; Stahl, B.; Strupat, K.; Hillenkamp, F. Anal. Chim. Acta 1990, 241, 175.

3. Beavis, R. C.; Chait, B. T. Rapid Commun. Mass Spectrom. 1989, 3, 432.

4. Hillenkamp, F.; Karas, M.; Beavis, R. C.; Chait, B. T. Anal. Chem. 1991, 63, 1193A.

5. Spengler, B.; Kirsch, D.; Kaufmann, R. Rapid Commun. Mass Spectrom. 1991, 5, 198.

6. Karas, M.; Bahr, U.; Strupat, K.; Hillenkamp, F.; Tsarbopoulos, A.; l'ramanik, B. N. Anal. Chem. 1995, 67, 675.

7. Hill, J. A.; Annan, R. S.; Biemann, K. Rapid Commun. Mass Spectrom. 1991, 5, 395.

8. Spengler, B.; Kaufmann, R. Analysis 1992, $20,91$.

9. Kaufmann, R.; Kirsch, D.; Spengler, B. Int. J. Mass Spectrom. Ion Processes 1994, 131, 355.
10. Nordhoff, E.; Karas, M.; Cramer, R.; Hahner, S.; Hillenkamp, F.; Kirpekar, F.; Lexius, A.; Muth, J.; Meier, C.; Engels, J. W. J. Mass Spectrom. 1995, 30, 99.

11. Juhasz, P.; Costello, C. E. J. Am. Soc. Mass Spectrom. 1992, 3, 785.

12. Talbo, G.; Roepstorff, P. Rapid Commun. Mass Spectrom. 1993, 7, 201.

13. Spengler, B.; Kirsch, D.; Kaufmann, R.; Jaeger, E. Rapid Commun. Mass Spectrom. 1992, 6, 105.

14. Castoro, J. A.; Wilkins, C. L. Anal. Chem. 1993, 65, 2621.

15. Castoro, J. A.; Köster, C.; Wilkins, C. L. Rapid Commun. Mass Spectrom. 1992, 6, 239.

16. Tang, X.; Ens, W.; Standing, K. G.; Westmore, J. B. Anal. Chem. 1988, 60, 1791.

17. Tang, X.; Ens, W.; Mayer, F.; Standing, K. G.; Westmore, J. B. Rapid Commun. Mass Spectrom. 1989, 3, 443.

18. Brown, R. S.; Lennon, J. J. Anal. Chem. 1995, 67, 1998.

19. Brown, R. S.; Lennon, J. J. Anal. Chem. 1995, 67, 3990.

20. Karas, M.; Bahr, U.; Giessman, U. Mass Spectrom. Rev. 1991, 10, 355.

21. Brown, R. S.; Gilfrich, N. L. Anal. Chim. Acta 1991, $248,541$.

22. Beavis, R. C.; Chait, B. T. Anal. Chem. 1990, 62, 1836.

23. Johnson, R. S.; Martín, S. A.; Biemann, K. Int. I. Mass Spectrom. Ion Processes 1988, 86, 137.

24. Lee, H.; Lubman, D. M. Anal. Chem. 1995, 67, 1400 ,

25. Kinsel, G. R.; Lindner, J.; Grotemeyer, J.; Schlag, E. W. Proceedings of the 39th ASMS Conference on Mass Spectrometry and Allied Topics; Nashville, TN, May 19-24, 1991; pp 350.

26. Beavis, R. C.; Chaudhary, T.; Chait, B. T. Org. Mass Spectrom. 1992, 27, 156.

27. Patterson, S. D.; Katta, V. Anal. Chem. 1994, 66, 3727.

28. Crimmins, D. L.; Saylor, M.; Rush, J.; Thoma, R. S. Anal. Biochem. 1995, 226, 355. 